Tohoku J. Exp. Med., 2009, 218, 17-24

\title{
Factors Determining Cardiovascular and Renal Outcomes after Adrenalectomy in Patients with Aldosterone-Producing Adrenal Adenoma
}

\author{
Terry Ting-Yu Chiou, ${ }^{1}$ Po-Hui Chiang, ${ }^{2}$ Morgan Fuh, ${ }^{3}$ Rue-Tsuan Liu, \\ Wei-Ching Lee, ${ }^{2}$ Wen-Chin Lee, ${ }^{1}$ Hwee-Yeong Ng, ${ }^{1}$ Yu-Che Tsai, ${ }^{1}$ \\ Fong-Rong Chuang, ${ }^{1}$ Chao-Cheng Huang ${ }^{5}$ and Chien-Te Lee ${ }^{1}$



Primary aldosteronism is an important cause of secondary hypertension, because it is potentially curable, especially in case of unilateral aldosterone-producing adrenal adenoma (APA). However, the information is limited concerning the cardiovascular and renal outcomes in this patient population. We studied 52 patients with APA in order to determine the pre-operative and post-operative factors predicting cardiovascular and renal outcomes. All 52 patients were hypertensive before the operation. Among 35 patients who underwent pre-operative electrocardiogram, 23 patients had left ventricular hypertrophy (LVH). Patients with LVH had lower estimated glomerular filtration rate (eGFR). Adrenalectomy successfully normalized or improved hypertension, hypokalemia, and aldosterone excess. One month after the adrenalectomy, 32 patients $(62 \%)$ became normotensive, but 20 patients $(38 \%)$ remained hypertensive. However, after an average follow-up period of 51 months, only 18 patients remained normotensive, while 34 patients were hypertensive. Thus, the rate of recurrent hypertension after adrenalectomy was high (14/32, 43\%). Preoperative systolic blood pressure (BP), diastolic BP, and post-operative plasma aldosterone concentrations were the only variables significantly different between the hypertensive and normotensive patients. Using pre-operative BP $165 / 110 \mathrm{mmHg}$ as a cutoff has good positive predictive values (73-92\%) for post-operative long-term hypertension. Patients whose renal function worsened after adrenalectomy had significantly higher pre-operative plasma active renin levels. Thus, in patients with APA, the presence of LVH is correlated with impaired renal function (lower eGFR). In conclusion, pre-operative BP and post-operative plasma aldosterone are important in predicting post-adrenalectomy hypertension, and a lower pre-operative plasma renin predicts the improvement in renal function after adrenalectomy. —— Aldosteronism; adrenal adenoma; blood pressure; renal function; LVH.

Tohoku J. Exp. Med., 2009, 218 (1), 17-24. (C) 2009 Tohoku University Medical Press

Primary aldosteronism (PA) is an important cause of secondary hypertension because it may be curable. PA implies autonomous hypersecretion of aldosterone, which has been shown to cause damage to vital tissues or organs such as heart and vasculatures. (Milliez et al. 2005; Holaj et al. 2007). Its classical features include hypertension, hypokalemia and metabolic alkalosis. Recently, the wide use of aldosterone-renin ratio (ARR) as a screening test in hypertensive patients has led to a 5 - to 15 -fold increase in the detection of PA (Mulatero et al. 2004). The reported prevalence of PA ranges from $2 \%$ to $11.2 \%$ in hypertensive population (Lund et al. 1981; Young 1999; Rossi et al. 2006a; Fogari et al. 2007), while the prevalence in normotensive subjects is about $1 \%$ (Gordon 1994; Brown et al. 1996; Mosso et al. 2003). The two most common etiologies of primary aldosteronism are aldosterone-producing adrenal

Received November 26, 2008; revision accepted for publication March 19, 2009.

Correspondence: Chien-Te Lee, MD, PhD, Division of Nephrology, Department of Internal Medicine, Chang Gung Memorial Hospital-

Kaohsiung Medical Center, 123 Ta-Pei Road, Niao-Sung Hsiang, Kaohsiung Hsien 833, Taiwan.

e-mail: chientel@gmail.com, ctlee33@adm.cgmh.org.tw 
adenoma (APA) and bilateral adrenal hyperplasia. The other less common etiologies include unilateral adrenal hyperplasia, glucocorticoid-remediable hyperaldosteronism, aldosterone-producing adrenal carcinoma, and bilateral aldosterone-producing adenomas (Wu et al. 2008). In general, the treatment of choice for APA is surgical adrenalectomy. Although surgical adrenalectomy may cure hypertension in some patients with APA, as many as 30 70\% of the patients have persistent hypertension post-operatively (Blumenfeld et al. 1994; Foo et al. 2001; Meyer et al. 2005).

In addition to hypertension, growing literatures have recognized the important role of aldosterone in end organ damage, including heart, kidney and brain (Connell et al. 2008; Rayner 2008). We retrospectively analyze the clinical and biochemical parameters of patients with APA in our medical center in order to determine the factor(s) contributing to hypertension and cardiovascular and renal outcomes.

\section{Materials and Methods}

We searched our hospital records from 1987 to 2006 for patients who underwent adrenalectomy and had adrenal adenoma proven by pathology. Those patients with biochemical evidence of primary aldosteronism were included. Biochemical evidence of PA includes elevated serum aldosterone concentration greater than $162 \mathrm{pg} / \mathrm{mL}$, suppressed plasma active renin level less than $2.4 \mathrm{pg} / \mathrm{mL}$, and elevated aldosterone-to-renin ratio greater than 30 . Suspected patients were further investigated with imaging studies including computed tomography (CT) scan or NP-59 adrenal scan in order to help determine a lateralizing adrenal lesion. In patients whose CT or adrenal scans were equivocal, adrenal vein sampling was performed to distinguish the subtypes of PA. Patients with known history of primary renal disease or nephrectomy were excluded from our analysis because of the potential confounding effect on patients' renal and cardiovascular outcomes. A total of 52 patients were confirmed to have aldosteroneproducing adrenal adenoma, and were included for this study.

The medical records of these patients were carefully reviewed, including the initial presenting signs and symptoms, duration of disease (from onset of hypertension to operation), serum creatinine and proteinuria, blood pressure (BP), left ventricular hypertrophy (LVH), and cerebrovascular accidents. Antihypertensive medications before and after adrenalectomy were recorded: the classes and total number used by each patient. The medication classes included aldosterone antagonist, diuretic, calcium channel blocker, angiotensin-converting enzyme inhibitors, angiotensin receptor blockers, $\beta$-blockers, and $\alpha$-blockers. Pre-operative BP was measured by the standard mercury sphygmomanometer in the physician's office. Patients were maintained on their usual antihypertensive medications. The average of at least ten office measurements was recorded. The pre-operative electrocardiographic LVH is based on: $\mathrm{S}$ in lead V3 plus $\mathrm{R}$ in aVL $>2.8$ $\mathrm{mV}$ for men, $\mathrm{S}$ in lead $\mathrm{V} 3+\mathrm{R}$ in $\mathrm{aVL}>2.0 \mathrm{mV}$ for women.

Serum aldosterone was measured by DSL-8600 ACTIVE Aldosterone Coated-Tube Radioimmunoassay kit (Diagnostic System Laboratories, Inc. Webster, Texas USA). Plasma active renin was measured by RENIN III GENERATION radioimmunometric assay kit (CIS bio international, France). Glomerular filtration rate (eGFR) was estimated using the abbreviated Modification of Diet in Renal Disease Study Group (MDRD) equation (Levey et al. 1999): eGFR $=175 \times$ Creatinine $\mathrm{e}^{-1.154} \times \mathrm{Age}^{-0.203}(\times 1$ if male, $\times 0.742$ if female, $\times 1.21$ if black). This calculation takes into consideration serum creatinine, age, gender, and race. Pre-operative eGFR was calculated using the serum creatinine obtained right before adrenalectomy, and post-operative eGFR was calculated using the most recent serum creatinine data available after surgery. Proteinuria is diagnosed with routine urine dipstick test.

Patients were divided into two groups according to the postoperative BP levels. Comparisons between these two groups were made at two different time points. We chose one month after the operation as the first time point in order to observe the immediate effect of adrenalectomy. The second time point was the latest hospital follow-up visit available on medical record, which would provide the long-term outcome of these patients. Patients were classified as normotensive if their BP was less than 140/90 $\mathrm{mmHg}$ without antihypertensive treatment. Patients taking antihypertensive medications or with BP of greater than $140 / 90 \mathrm{mmHg}$ were classified as hypertensive.

The statistical analysis was performed using SPSS software, version 12.0. In this report, continuous variables are expressed as mean ( \pm standard deviation) unless otherwise indicated. Patient characteristics before and after adrenalectomy were compared using paired $t$ test (if variables follow a normal distribution) or Wilcoxon Signed Ranks test (if variables are not in normal distribution). Variables between different subgroups were compared using unpaired $t$ test (if variables follow a normal distribution) or Mann-Whitney test (if variables are not in normal distribution). Pearson's correlation test was used to determine the correlations between different variables. In order to identify the optimal cutoffs of predictors for hypertension, we used the area under the curve (AUC) of receiver operator characteristics (ROC) analysis. The value with optimum sensitivity and specificity was chosen.

\section{Results}

The mean age of our 52 patients with APA was $43 \pm 10$ (range 22-66) years old, and there were 31 female and 21 male patients. The mean duration of disease was $6 \pm 5.4$ yeas (range 1 month-28 years). Thirty-five of the 52 patients had the adrenal adenoma on their left side.

The most common initial presenting sign or symptom was hypertension (69\%), followed by general or limb weakness $(38 \%)$. There were two patients presenting with stroke and one with chest pain. Cardiology clinic is the most common department for initial presentation (46\%), followed by nephrology (21\%), emergency room (8\%), urology (8\%), endocrinology (6\%), neurology (2\%), and family medicine $(2 \%)$. Hypokalemia (serum potassium $<3 \mathrm{meq} / \mathrm{L}$ ) was documented in 48 patients (92\%).

The average BP and biochemical profiles before and after adrenalectomy are shown in Table 1. All patients were hypertensive before adrenalectomy. Average BP and potassium were improved significantly after surgery. Estimated GFR did not change significantly. However, average serum aldosterone level, plasma active renin, aldosterone to renin ratio (ARR), and number of antihypertensive medications all changed significantly after adrenalectomy.

\section{Vascular complications}

Twenty-three of the 35 patients (65\%) who had pre- 
Table 1. Patients' blood pressures and biochemical profiles before and after adrenalectomy.

\begin{tabular}{|c|c|c|c|}
\hline & $\begin{array}{l}\text { Before adrenalectomy } \\
\qquad(N=52)\end{array}$ & $\begin{array}{l}\text { After adrenalectomy } \\
\qquad(N=52)\end{array}$ & $p$-value \\
\hline Systolic BP (mmHg) & $192 \pm 24$ & $142 \pm 20$ & $<0.001$ \\
\hline Diastolic BP(mmHg) & $113 \pm 15$ & $89 \pm 13$ & $<0.001$ \\
\hline Serum potassium $(\mathrm{meq} / \mathrm{L})$ & $2.3 \pm 0.5$ & $4.1 \pm 0.7$ & $<0.001$ \\
\hline eGFR $\left(\mathrm{ml} / \mathrm{min} / 1.73 \mathrm{~m}^{2}\right)$ & $74.8 \pm 22.7$ & $66.8 \pm 21.7$ & 0.064 \\
\hline Plasma aldosterone concentration (pg/mL) & $754 \pm 422$ & $104 \pm 134$ & $<0.001$ \\
\hline Plasma active renin $(\mathrm{pg} / \mathrm{mL})$ & $8.9 \pm 9.8$ & $75 \pm 197$ & 0.008 \\
\hline Aldosterone:renin ratio & $834 \pm 1851$ & $116 \pm 285$ & 0.002 \\
\hline Number of Antihypertensive medications & $3.0 \pm 1.5$ & $1.2 \pm 1.2$ & $<0.001$ \\
\hline
\end{tabular}

BP, Blood pressure; eGFR, estimated glomerular filtration rate.

Table 2. Comparison of clinical features between hypertensive and normotensive patients one month after adrenalectomy.

\begin{tabular}{|c|c|c|c|}
\hline & $\begin{array}{l}\text { Hypertensive } \\
\quad(N=20)\end{array}$ & $\begin{array}{l}\text { Normotensive } \\
\quad(N=32)\end{array}$ & $P$-value \\
\hline Age (years old) & $43.1 \pm 11.7$ & $43.1 \pm 10.0$ & 0.985 \\
\hline Duration of disease (years) & $6.8 \pm 6.3$ & $5.5 \pm 4.9$ & 0.446 \\
\hline Pre-operative SBP (mmHg) & $203 \pm 21$ & $185 \pm 23$ & 0.007 \\
\hline Pre-operative DBP (mmHg) & $119 \pm 15$ & $108 \pm 14$ & 0.008 \\
\hline Post-operative SBP (mmHg) & $157 \pm 14$ & $132 \pm 17$ & $<0.001$ \\
\hline Post-operative DBP (mmHg) & $96 \pm 14$ & $84 \pm 11$ & 0.001 \\
\hline Pre-operative Serum Potassium (meq/L) & $2.4 \pm 0.4$ & $2.3 \pm 0.6$ & 0.692 \\
\hline Post-operative Serum Potassium (meq/L) & $4.2 \pm 0.7$ & $4.1 \pm 0.8$ & 0.610 \\
\hline Pre-operative eGFR (ml/min/1.73m²) & $73.9 \pm 20.7$ & $78.6 \pm 20.9$ & 0.433 \\
\hline Post-operative eGFR (ml/min/1.73m²) & $63.5 \pm 23.6$ & $71.1 \pm 22.7$ & 0.417 \\
\hline Change in eGFR $\left(\mathrm{ml} / \mathrm{min} / 1.73 \mathrm{~m}^{2}\right)$ & $-0.5 \pm 14.7$ & $-10 \pm 26.5$ & 0.300 \\
\hline Pre-operative Plasma Aldosterone (pg/mL) & $671.6 \pm 372.6$ & $753.6 \pm 434.4$ & 0.503 \\
\hline Pre-operative Plasma Renin (pg/mL) & $11.1 \pm 9.9$ & $9.1 \pm 10.7$ & 0.531 \\
\hline Pre-operative ARR & $553.9 \pm 1042$ & $677.2 \pm 1521$ & 0.772 \\
\hline Post-operative Plasma Aldosterone (pg/mL) & $125.5 \pm 154.1$ & $77.6 \pm 105.4$ & 0.288 \\
\hline Post-operative Plasma Renin (pg/mL) & $129.1 \pm 277.8$ & $21.8 \pm 28.5$ & 0.329 \\
\hline Post-operative ARR & $15.3 \pm 21.4$ & $217.2 \pm 389.7$ & 0.220 \\
\hline Number of Pre-operative Antihypertensive medications & $3.2 \pm 1.8$ & $2.8 \pm 1.3$ & 0.418 \\
\hline Number of Post-operative Antihypertensive medications & $1.5 \pm 1.4$ & $1.1 \pm 1.2$ & 0.360 \\
\hline
\end{tabular}

SBP, systolic blood pressure; DBP, diastolic blood pressure; eGFR, estimated glomerular filtration rate; ARR, Aldosterone-Renin Ratio.

operative electrocardiogram were diagnosed with left ventricular hypertrophy (LVH). Seven patients $(13 \%)$ suffered from eight cerebrovascular accidents (CVA: four infarctions, one hemorrhagic, and three unknown). Four CVA occurred before adrenalectomy (4 months to 9 years), and another four occurred after surgery (2 days to 7 years).

When grouping the 35 patients according to the presence of pre-operative LVH, those with LVH had significantly lower pre-operative eGFR and post-operative eGFR than those without LVH (66.8 vs. $87.6 \mathrm{~mL} / \mathrm{min} / 1.73 \mathrm{~m}^{2}, p=0.006$; 54.3 vs. $76.5 \mathrm{~mL} / \mathrm{min} / 1.73 \mathrm{~m}^{2}, p=0.017$, respectively). Duration of disease came close to statistical significance $(p$ $=0.051,7.7$ years in LVH group vs. 3.5 years in non-LVH group). These two groups did not differ significantly in oth- er parameters such as BP, serum potassium level, plasma aldosterone concentration, plasma active renin, or proteinuria.

At one month after adrenalectomy, 32 patients $(62 \%)$ were normotensive, and 20 patients $(38 \%)$ were hypertensive. Between the two groups (Table 2), pre-operative systolic BP and diastolic BP were the only variables that showed significant difference ( $p=0.007$ and 0.008 , respectively). The numbers of pre-operative antihypertensive medications were not different between the two groups. Variables such as age, serum potassium level, duration of disease, plasma aldosterone concentration, plasma active renin, and ARR were not significantly different between the two groups. 
Table 3. Comparison of clinical features between hypertensive and normotensive patients after adrenalectomy on long term follow-up.

\begin{tabular}{|c|c|c|c|}
\hline & $\begin{array}{c}\text { Hypertensive } \\
(N=34)\end{array}$ & $\begin{array}{l}\text { Normotensive } \\
\quad(N=18)\end{array}$ & $P$-value \\
\hline Age (years old) & $42.3 \pm 10.7$ & $44.6 \pm 10.3$ & 0.453 \\
\hline Duration of disease (years) & $6.0 \pm 5.5$ & $6.0 \pm 4.9$ & 0.989 \\
\hline Pre-operative SBP (mmHg) & $199 \pm 21$ & $178 \pm 21$ & 0.002 \\
\hline Pre-operative DBP (mmHg) & $116 \pm 15$ & $105 \pm 10$ & 0.006 \\
\hline Post-operative SBP (mmHg) & $147 \pm 17$ & $130 \pm 20$ & 0.002 \\
\hline Post-operative DBP (mmHg) & $91 \pm 13$ & $84 \pm 12$ & 0.053 \\
\hline Pre-operative Serum Potassium (meq/L) & $2.3 \pm 0.4$ & $2.3 \pm 0.5$ & 0.889 \\
\hline Post-operative Serum Potassium (meq/L) & $4.1 \pm 0.7$ & $4.1 \pm 0.7$ & 0.903 \\
\hline Pre-operative eGFR $\left(\mathrm{ml} / \mathrm{min} / 1.73 \mathrm{~m}^{2}\right)$ & $76.0 \pm 2.2$ & $78.2 \pm 18.1$ & 0.721 \\
\hline Post-operative eGFR $\left(\mathrm{ml} / \mathrm{min} / 1.73 \mathrm{~m}^{2}\right)$ & $68.3 \pm 24.1$ & $65.6 \pm 19.8$ & 0.820 \\
\hline Change in eGFR $\left(\mathrm{ml} / \mathrm{min} / 1.73 \mathrm{~m}^{2}\right)$ & $1.65 \pm 0.48$ & $1.2 \pm 0.44$ & 0.075 \\
\hline Pre-operative Plasma Aldosterone (pg/mL) & $690.6 \pm 376.2$ & $782.1 \pm 474.9$ & 0.471 \\
\hline Pre-operative Plasma Renin (pg/mL) & $9.7 \pm 8.7$ & $10.1 \pm 13.8$ & 0.902 \\
\hline Pre-operative ARR & $480.2 \pm 860.5$ & $970.5 \pm 2075.6$ & 0.425 \\
\hline Post-operative Plasma Aldosterone (pg/mL) & $125.0 \pm 148.1$ & $46.2 \pm 43.2$ & 0.022 \\
\hline Post-operative Plasma Renin (pg/mL) & $60.8 \pm 128.3$ & $15.0 \pm 21.1$ & 0.635 \\
\hline Post-operative ARR & $93.8 \pm 284.4$ & $250.9 \pm 352.1$ & 0.493 \\
\hline Number of Pre-operative Antihypertensive medications & $3.2 \pm 1.5$ & $2.5 \pm 1.4$ & 0.144 \\
\hline Number of Post-operative Antihypertensive medications & $1.7 \pm 1.2$ & 0 & 0.000 \\
\hline
\end{tabular}

SBP, systolic blood pressure; DBP, diastolic blood pressure; eGFR, estimated glomerular filtration rate; ARR, Aldosterone-Renin Ratio

After an average follow-up period of 51 months, only 18 patients $(35 \%)$ remained normotensive, and 34 patients $(65 \%)$ were hypertensive (Table 3 ). Between the two groups, significant differences were found in their postoperative plasma aldosterone concentrations, and pre-operative systolic BP and diastolic BP. The numbers of antihypertensive medications used before adrenalectomy were not different between these two groups. In addition, the proportions of patients using different classes of antihypertensive medications were not different between the two groups (data not shown). Other variables such as age, serum potassium level, duration of disease, plasma active renin, and ARR were not significantly different between the two groups. Using pre-operative systolic BP of $165 \mathrm{mmHg}$ as a cutoff value has a positive predictive value of $73 \%$ and a negative predictive value of $85 \%$ for predicting post-adrenalectomy hypertension or normotension on the long-term follow-up. Similarly, a cutoff level of $110 \mathrm{mmHg}$ for pre-operative diastolic BP can have a positive predictive value of $92 \%$ and a negative predictive value of $57 \%$. Moreover, a post-operative plasma aldosterone level of $187 \mathrm{pg} / \mathrm{mL}$ can have a positive predictive value of $100 \%$ and a negative predictive value of $40 \%$. The ROC curves for systolic BP, diastolic BP and plasma aldosterone concentration predicting post-adrenalectomy hypertension are shown in Fig. 1. Of the 32 patients who were normotensive at one month after adrenalectomy, 14 (43\%) had recurrent hypertension at the longterm follow-up. Patients with recurrent hypertension were not significantly different from those who remained normotensive in the demographic and laboratory variables tested in this study.

\section{Renal outcome}

The mean pre-operative eGFR of all patients was 76.8 $\pm 2.9 \mathrm{~mL} / \mathrm{min} / 1.73 \mathrm{~m}^{2}$. In the 35 patients who had long-term follow-up renal function (mean 51 months, range 1-175 months) after surgery, mean eGFR changed from 74.8 $( \pm 22.7)$ to $66.8( \pm 21.7) \mathrm{mL} / \mathrm{min} / 1.73 \mathrm{~m}^{2}(p=0.064)$. In the 25 patients with serum creatinine measured within one year after adrenalectomy, mean eGFR changed from 73.6 $( \pm 23.3)$ to $67.7( \pm 22.9) \mathrm{mL} / \mathrm{min} / 1.73 \mathrm{~m}^{2}(p=0.201)$. However, when grouping the 25 patients according to the change of eGFR within one year after surgery, those with decreased eGFR $(N=14$, mean change in eGFR $-21.1 \pm$ $16.4 \mathrm{~mL} / \mathrm{min} / 1.73 \mathrm{~m}^{2}$ ) had significantly higher pre-operative plasma active renin $(12.7 \pm 9.4 \mathrm{pg} / \mathrm{mL})$ compared to those with eGFR not decreased $(N=7$, mean change $+13.6 \pm 9.4$ $\mathrm{mL} / \mathrm{min} / 1.73 \mathrm{~m}^{2}$, plasma active renin $4.3 \pm 3.6 \mathrm{pg} / \mathrm{mL}, p=$ $0.009)$. The mean pre-operative eGFR did not differ significantly between these two groups of patients $(65.4 \pm 21.5 \mathrm{vs}$. $\left.80.1 \pm 23.3 \mathrm{~mL} / \mathrm{min} / 1.73 \mathrm{~m}^{2}, p=0.119\right)$. These two groups did not differ significantly in other parameters such as age, duration of disease, BP, plasma aldosterone concentration, or presence of $\mathrm{LVH}$.

Of the 43 patients who had routine urinalysis performed before adrenalectomy, $16(37 \%)$ had proteinuria. 
a

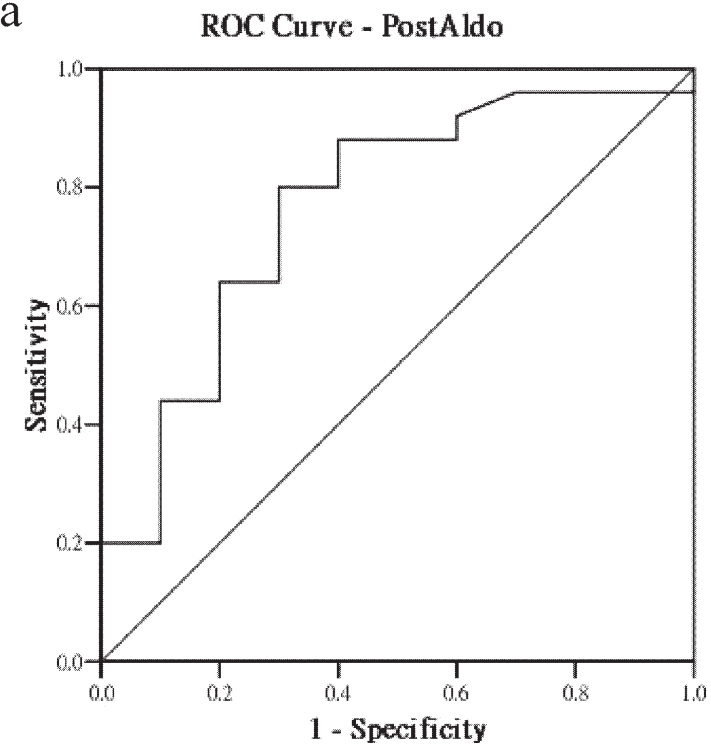

b

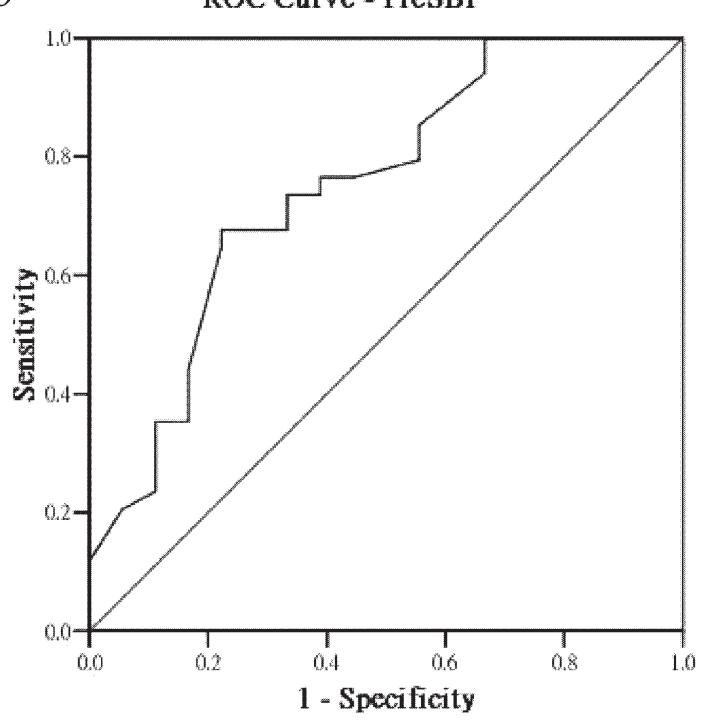

After surgery, only 17 patients had routine urinalysis, and 5 of them had proteinuria. When grouping the 43 patients according to the presence of pre-operative proteinuria, those with proteinuria had lower pre-operative serum potassium than those without $(2.0$ vs. $2.5 \mathrm{meq} / \mathrm{L}, p=0.001)$. These two groups did not differ significantly in other parameters such as age, duration of disease, BP, eGFR, plasma aldosterone concentration, plasma active renin, or presence of LVH.

\section{Correlation study}

The correlation study found that the duration of disease is correlated positively with age $(r=0.626, p<0.001)$ and negatively with pre-operative serum potassium level ( $r=$ $-0.300, p=0.034)$. Age is correlated positively with preoperative aldosterone-renin ratio $(r=0.392, p=0.009)$, and negatively with pre-operative eGFR $(r=-0.430, p=0.001)$, post-operative eGFR $(r=-0.554, p=0.004)$ and post-oper-
$\mathrm{C}$

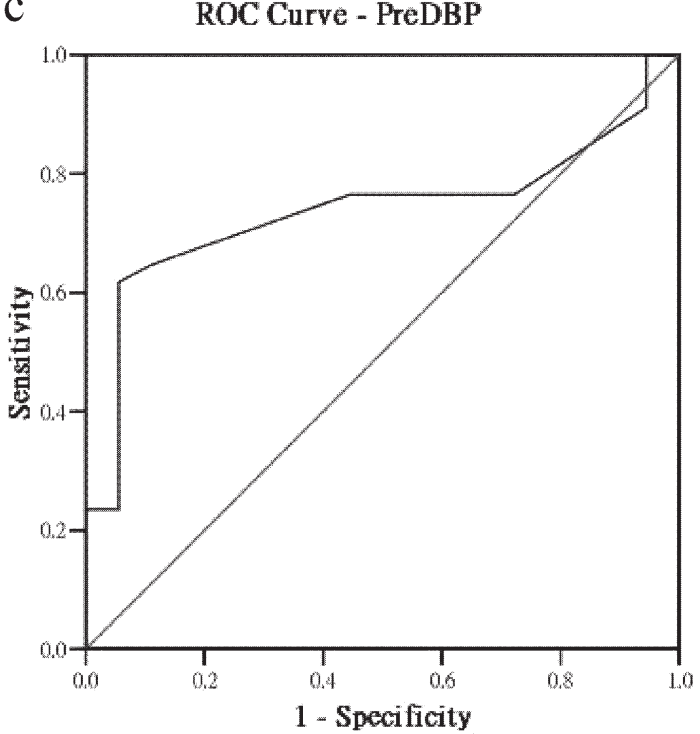

Fig. 1. The ROC curves for plasma aldosterone concentration, systolic BP, and diastolic BP predicting post-adrenalectomy hypertension.

(a) ROC curve for post-operative plasma aldosterone concentration predicting post-adrenalectomy hypertension (Area under the ROC curve $=0.766, P=0.015$ ).

(b) ROC curve for pre-operative systolic blood pressures (SBP) predicting post-adrenalectomy hypertension (Area under the ROC curve $=0.749, P=0.003$ ).

(c) ROC curve for pre-operative diastolic blood pressures (DBP) predicting post-adrenalectomy hypertension (Area under the ROC curve $=0.738, P=0.005$ ).

ative diastolic BP $(r=-0.324, p=0.019)$. Pre-operative eGFR is correlated negatively with duration of disease ( $r=$ $-467, p=0.001)$, but positively with post-operative eGFR $(r=0.539, p=0.005)$. Pre- and post-operative aldosterone and post-operative renin are not significantly correlated with any of the other parameters. BP is not correlated with age, duration of disease, aldosterone, renin, ARR, or eGFR.

\section{Discussion}

End-organ damage due to PA has been demonstrated in the previous literatures. The most common complications include hypertension and adverse cardiovascular outcomes such as myocardial infarction, stroke, atrial fibrillation, and vascular remodeling (Milliez et al. 2005; Catena et al. 2007; Holaj et al. 2007; Catena et al. 2008; Rossi et al. 2008). The prevalence of these cardiovascular events is greater in PA than in essential hypertension. In patients with APA, hypertension may be improved or even cured by adrenalec- 
tomy. Such clinical improvement is usually accompanied by the normalization of serum potassium and plasma aldosterone concentration (Omura et al. 2006). However, a proportion of the patients with APA (30-70\%) may have persistent hypertension (Blumenfeld et al. 1994; Foo et al. 2001; Meyer et al. 2005) or still require anti-hypertensive medications after adrenalectomy (Obara et al. 1992; Celen et al. 1996; Proye et al. 1998). This may be caused by concomitant essential hypertension or irreversible vascular damage from exposure to high BP or aldosterone excess. Several previously reported risk factors for persistent hypertension include old age, duration of disease, family history, and use of more than two anti-hypertensive agents (Fronticelli et al. 1995; Horita et al. 2001; Sawka et al. 2001).

Our study examined both the immediate and long-term effects of adrenalectomy in patients with APA. At one month post-operatively, 32 patients $(62 \%)$ became normotensive. At follow-up, all 20 patients who were hypertensive at one month remained hypertensive, and 14 of the 32 patients who were previously normotensive at one month had recurrent hypertension. The rate of recurrent hypertension in our series was $43 \%$, which is compatible with previous reports (Obara et al. 1992; Blumenfeld et al. 1994; Celen et al. 1996; Fukudome et al. 2002). Furthermore, we found that pre-operative BP is the only predictor for postoperative hypertension at one month, and pre-operative BP and post-operative plasma aldosterone concentration are the important predictors for hypertension at the long-term follow-up. This suggests that in the initial post-operative period, patients with lower pre-operative BP are more likely to become normotensive. However, at follow-up, more patients will become hypertensive, and this is associated with higher levels of pre-operative BP and post-operative plasma aldosterone. This increase in the proportion of hypertensive patients at follow-up can result from aging, development of essential hypertension and irreversible vascular damage by long-standing high BP and aldosterone excess. In the report by Zarnegar et al. (2008), post-operative aldosterone also emerged as one of the several distinguishing factors between patients with resolved and not resolved hypertension.

Our predictive values based on pre-operative BP and post-operative plasma aldosterone concentration are comparable to the Aldosteronoma Resolution Score (ARS) model (negative predictive value $72.4-86.3 \%$ and positive predictive value $75-80 \%$ ), reported by Zarnegar et al. (2008). The ARS model uses four pre-operative parameters to predict resolution of hypertension, including female, years of hypertension $\leq 6$, body mass index $\leq 25$, and number of anti-hypertensive medications $\leq 2$. Similarly, Lumachi et al. (2005) found that the age of patients and duration of hypertension may have a maximum predictive power of $75 \%$ for unresolved hypertension. Moreover, Fukudome et al. (2002) demonstrated in a multivariate analysis that only pre-operative serum creatinine was independently associated with recurrent hypertension. One of the major differenc- es among these studies is the duration of follow-up period: 6 months in ARS model (Zarnegar et al. 2008), 81 months (Lumachi et al. 2005), 12.2 years (Fukudome et al. 2002), and 51 months in the present study. These findings suggest that different factors have variable importance at different follow-up periods. In addition, our study used eGFR, which is more representative of renal function than serum creatinine alone. Unfortunately, the variation in eGFR is large and no significant difference was detected between the hypertensive and normotensive groups. Our model offers another set of pre-operative parameters to help clinicians predict post-adrenalectomy hypertension, which may be associated with significant long-term morbidity and healthcare cost.

In addition to the cardiovascular system, primary aldosteronism can affect the kidney function (Ribstein et al. 2005; Rossi et al. 2006b; Sechi et al. 2006). Ribstein et al. (2005) and Sechi et al. (2006) compared patients with primary aldosteronism (including both APA and bilateral adrenal hyperplasia) to those with essential hypertension. They suggested that PA is characterized by relative glomerular hyperfiltration and albuminuria, both of which may be reduced after removal of aldosterone excess, either by adrenalectomy or aldosterone antagonist. The prevalence of pre-operative proteinuria $(37 \%)$ is high in our patients. Mean pre-operative eGFR of our patients was $74.8 \mathrm{ml} /$ $\mathrm{min} / 1.73 \mathrm{~m}^{2}$, and we did not find any significant change in eGFR (i.e. no relative glomerular hyperfiltration) after adrenalectomy. Our finding is similar to the report by Rossi et al. (2006b), which suggested that glomerular hyperfiltration may not be a hallmark of PA. Our study differs from these studies in several aspects. We did not exclude patients with renal dysfunction, and methods for estimating eGFR are different. Our patients are younger and have much higher mean plasma aldosterone concentrations, and higher mean BP. Furthermore, $92 \%$ of our patients were hypokalemic, a much higher proportion than that reported in the recent series of primary aldosteronism patients (Mulatero et al. 2004). Therefore, our cohort represents patients with a more severe disease and likely irreversible damage to the kidney.

One interesting finding from our analysis is that patients whose eGFR improved after adrenalectomy had lower pre-operative plasma renin than those with decreased eGFR after the operation. In patients with eGFR improved after adrenalectomy, aldosterone excess was the major cause of renal function impairment. As expected, plasma renin level was suppressed by the elevated aldosterone. However, in patients with decreased eGFR after the operation, plasma renin is higher, not completely suppressed by the elevated aldosterone. This suggests the coexistence of a high aldosterone state due to the adenoma and a nonsuppressable renin state from other causes, such as essential hypertension. This nonsuppressable renin state would result in additional kidney damage that cannot be reversed by adrenalectomy. Moreover, this change in eGFR is not correlated with 
age, duration of disease, BP, or plasma aldosterone concentration. These findings suggest that patients with APA are heterogeneous in regards to their mechanisms for impaired eGFR. And according to our analysis, worsening eGFR may be related to the nonsuppressable plasma renin. In addition to the change in eGFR, proteinuria is another marker of kidney dysfunction. Our finding that patients with proteinuria had lower pre-operative serum potassium than those without proteinuria is similar to that reported by Rossi et al. (2006b). Their cross-sectional study found a weak inverse relation between urinary albumin excretion (UAE) rate and serum potassium, suggesting a role for hypokalemia in raising UAE rate. The coexistence of proteinuria and hypokalemia also suggests simultaneous injury to both the glomeruli and renal tubules.

Another interesting finding is the strong association between cardiac and renal outcomes. We found that patients with APA and LVH had lower eGFR, compared to those without LVH. However, neither LVH nor eGFR are significantly correlated with BP. The dysfunction of both heart and kidneys may be more closely related to other mechanisms, such as generalized endothelial dysfunction and increased oxidative stress (Goligorsky 2007; Zoccali 2007; Neade and Uribarri 2008).

Duration of disease is correlated with age and preoperative serum potassium. For older patients, physicians are more likely to attribute high BP to essential hypertension, thereby delaying search for secondary hypertension. The longer the presence of aldosterone excess, the more potassium depletion will occur. The negative correlation between age and eGFR may reflect the natural course of aging.

Our study has several potential limitations. First, this study is a retrospective data analysis. Some of the patients were not followed up regularly at our hospital after surgery, leading to incomplete data in the long-term follow-up period. Because we are a tertiary medical center, a large proportion of these APA patients were referred from local clinics or hospitals due to refractory hypertension. These patients may represent the more clinically severe group, and inference to the general population of patients with aldosteronoma should be done with cautions. In addition, the limitation of using electrocardiogram alone to diagnose LVH should be acknowledged. Although the electrographic method is not as accurate as the echocardiographic method, it is more easily obtained and less costly.

In conclusion, primary aldosteronism due to aldosterone-producing adrenal adenoma is associated with significant cardiovascular and renal dysfunction. In the immediate post-adrenalectomy period, pre-operative BP is the only predictor for whether these patients will become normotensive or hypertensive. However, in the long-term, patients with higher pre-operative BP and post-operative plasma aldosterone concentrations are more likely to remain hypertensive after adrenalectomy. The presence of LVH heralds a mild impairment of renal function. We believe these can help clinicians provide objective information about treatment outcome to patients with APA before undergoing surgical intervention.

\section{References}

Blumenfeld, J.D., Sealey, J.E., Schlussel, Y., Sos, T.A., Atlas, S.A., Müller, F.B., Acevedo, R., Ulick, S. \& Laragh, J.H. (1994) Diagnosis and treatment of primary hyperaldosteronism. Ann. Intern. Med., 121, 877-885.

Brown, M.A., Cramp, H.A., Zammit, V.C. \& Whitworth, J.A. (1996) Primary hyperaldosteronism: a missed diagnosis in 'essential hypertensives'? Aust. N. Z. J. Med., 26, 533-538.

Catena, C., Colussi, G., Lapenna, R., Nadalini, E., Chiuch, A., Gianfagna, P. \& Sechi, L.A. (2007) Long-term cardiac effects of adrenalectomy or mineralocorticoid antagonists in patients with primary aldosteronism. Hypertension, 50, 911-918.

Catena, C., Colussi, G., Nadalini, E., Chiuch, A., Baroselli, S., Lapenna, R. \& Sechi, L.A. (2008) Cardiovascular outcomes in patients with primary aldosteronism after treatment. Arch. Intern. Med., 168, 80-85.

Celen, O., O’Brien, M.J., Melby, J.C. \& Beazley, R.M. (1996) Factors influencing outcome of surgery for primary aldosteronism. Arch. Surg., 131, 646-650.

Connell, J.M., McaKenzie, S.M., Freel, E.M., Fraser, R. \& Davies, E. (2008) A lifetime of aldosterone excess: long-term consequences of altered regulation of aldosterone production for cardiovascular function. Endocr. Rev., 29, 133-154.

Fogari, R., Preti, P., Zoppi, A., Rinaldi, A., Fogari, E. \& Mugellini, A. (2007) Prevalence of primary aldosteronism among unselected hypertensive patients: a prospective study based on the use of an aldosterone/renin ratio above 25 as a screening test. Hypertens. Res., 30, 111-117.

Foo, R., O’Shaughnessy, K.M. \& Brown, M.J. (2001) Hyperaldosteronism: recent concept, diagnosis, and management. Postgrad. Med.J., 77, 639-644.

Fronticelli, C.M., Ferrero, A., Quiriconi, F., Bargoni, A. \& Masenti, E. (1995) Primary hyperaldosteronism. Analysis of risk factors associated with persistent postoperative hypertension. Int. Surg., 80, 175-177.

Fukudome, Y., Fujii, K., Arima, H., Ohya, Y., Tsuchihashi, T., Abe, I. \& Fujishima, M. (2002) Discriminating factors for recurrent hypertension in patients with primary aldosteronism after adrenalectomy. Hypertens. Res., 25, 11-18.

Goligorsky, M.S. (2007) Frontiers in nephrology: viewing the kidney through the heart-endothelial dysfunction in chronic kidney disease. J. Am. Soc. Nephrol., 18, 2833-2835.

Gordon, R.D. (1994) Mineralcorticoid hypertension. Lancet, 344, 240-243.

Holaj, R., Zelinka, T., Wichterle, D., Petrák, O., Strauch, B. \& Widimský, J. Jr. (2007) Increased intima-media thickness of the common carotid artery in primary aldosteronism in comparison with essential hypertension. J. Hypertens., 25, 1451-1457.

Horita, Y., Inenaga, T., Nakahama, H., Ishibashi-Ueda, H., Kawano, Y., Nakamura, S., Horio, T., Okuda, N., Ando, M. \& Takishita, S. (2001) Cause of residual hypertension after adrenalectomy in patients with primary aldosteronism. Am. J. Kidney Dis., 37, 884-889.

Levey, A.S., Bosch, J.P., Lewis, J.B., Greene, T., Rogers, N. \& Roth, D. (1999) A more accurate method to estimate glomerular filtration rate from serum creatinine: a new prediction equation. Modification of Diet in Renal Disease Study Group. Ann. Intern. Med., 130, 461-470.

Lumachi, F., Ermani, M., Basso, S.M., Armanini, D., Iacobone, M. \& Favia, G. (2005) Long-term results of adrenalectomy in patients with aldosterone-producing adenomas: multivariate analysis of factors affecting unresolved hypertension and 
review of the literature. Am. Surg., 71, 864-869.

Lund, J., Nielsen, M. \& Giese, J. (1981) Prevalence of primary aldosteronism. Acta Med. Scand. Suppl., 646, 54-57.

Meyer, A., Brabant, G. \& Behrend, M. (2005) Long-term followup after adrenalectomy for primary aldosteronism. World $J$. Surg., 29, 155-159.

Milliez, P., Girerd, X., Plouin, P.F., Blacher, J., Safar, M.E. \& Mourad, J.J. (2005) Evidence for an increased rate of cardiovascular events in patients with primary aldosteronism. J. Am. Coll. Cardiol., 45, 1243-1248.

Mosso, L., Carvajal, C., Gonzalez, A., Barraza, A., Avila, F., Montero, J., Huete, A., Gederlini, A. \& Fardella, C.E. (2003) Primary aldosteronism and hypertensive disease. Hypertension, 42, 161-165.

Mulatero, P., Stowasser, M., Loh, K.C., Fardella, C.E., Gordon, R.D., Mosso, L., Gomez-Sanchez, C.E., Veglio, F. \& Young, W.F. Jr. (2004) Increased diagnosis of primary aldosteronism, including surgically correctable forms, in centers from five continents. J. Clin. Endocrinol. Metab., 89, 1045-1050.

Neade, T. \& Uribarri, J. (2008) Diet, inflammation, and chronic kidney disease: getting to the heart of the matter. Semin. Dial., 21, 331-337.

Obara, T., Ito, Y., Okamoto, T., Kanaji, Y., Yamashita, T., Aiba, M. \& Fujimoto, Y. (1992) Risk factors associated with postoperative persistent hypertension in patients with primary aldosteronism. Surgery, 112, 987-993.

Omura, M., Sasano, H., Saito, J., Yamaguchi, K., Kakuta, Y. \& Nishikawa, T. (2006) Clinical characteristics of aldosteroneproducing microadenoma, macroadenoma, and idiopathic hyperaldosteronism in 93 patients with primary aldosteronism. Hypertens. Res., 29, 883-889.

Proye, C.A., Mulliez, E.A., Carnaille, B.M., Lecomte-Houcke, M., Decoulx, M., Wémeau, J.L., Lefebvre, J., Racadot, A., Ernst, O., Huglo, D. \& Carré, A. (1998) Essential hypertension: first reason for persistent hypertension after unilateral adrenalectomy for primary aldosteronism? Surgery, 124, 1128-1133.

Rayner, B. (2008) Primary aldosteronism and aldosterone-associated hypertension. J. Clin. Pathol., 61, 825-831.

Ribstein, J., Du Cailar, G., Fesler, P. \& Mimran, A. (2005) Relative glomerular hyperfiltration in primary aldosteronism. J. Am. Soc. Nephrol., 16, 1320-1325.
Rossi, G.P., Bernini, G., Caliumi, C., Desideri, G., Fabris, B., Ferri, C., Ganzaroli, C., Giacchetti, G., Letizia, C., Maccario, M., Mallamaci, F., Mannelli, M., Mattarello, M.J., Moretti, A., Palumbo, G., Parenti, G., Porteri, E., Semplicini, A., Rizzoni, D., Rossi, E., Boscaro, M., Pessina, A.C., Mantero, F. \& PAPY Study Investigators. (2006a) A prospective study of the prevalence of primary aldosteronism in 1,125 hypertensive patients. J. Am. Coll. Cardiol., 48, 2293-2300.

Rossi, G.P., Bernini, G., Desideri, G., Fabris, B., Ferri, C., Giacchetti, G., Letizia, C., Maccario, M., Mannelli, M., Matterello, M.J., Montemurro, D., Palumbo, G., Rizzoni, D., Rossi, E., Pessina, A.C., Mantero, F. \& PAPY Study Participants. (2006b) Renal damage in primary aldosteronism: results of the PAPY Study. Hypertension, 48, 232-238.

Rossi, G.P., Bolognesi, M., Rizzoni, D., Seccia, T.M., Piva, A., Porteri, E., Tiberio, G.A., Giulini, S.M., Agabiti-Rosei, E. \& Pessina, A.C. (2008) Vascular remodeling and duration of hypertension predict outcome of adrenalectomy in primary aldosteronism patients. Hypertension, 51, 1366-1371.

Sawka, A.M., Young, W.F., Thompson, G.B., Grant, C.S., Farley, D.R., Leibson, C. \& van Heerden, J.A. (2001) Primary aldosteronism: factors associated with normalization of blood pressure after surgery. Ann. Intern. Med., 135, 258-261.

Sechi, L.A., Novello, M., Lapenna, R., Baroselli, S., Nadalini, E., Colussi, G.L. \& Catena, C. (2006) Long-term renal outcomes in patients with primary aldosteronism. JAMA., 295, 2638-2645

Wu, V.C., Chueh, S.C., Chang, H.W., Lin, W.C., Liu, K.L., Li, H.Y., Lin, Y.H., Wu, K.D. \& Hsieh, B.S. (2008) Bilateral aldosterone-producing adenomas: differentiation from bilateral adrenal hyperplasia. Q. J. M., 101, 13-22.

Young, W. (1999) Primary aldosteronism: a common and curable form of hypertension. Cardiol. Rev., 7, 207-214.

Zarnegar, R., Young, W.F. Jr., Lee, J., Sweet, M.P., Kebebew, E., Farley, D.R., Thompson, G.B., Grant, C.S., Clark, O.H. \& Duh, Q.Y. (2008) The aldosteronoma resolution score: predicting complete resolution of hypertension after adrenalectomy for aldosteronoma. Ann. Surg., 247, 511-518.

Zoccali, C. (2007) The endothelium as a target in renal diseases. $J$. Nephrol., 20 Suppl 12, S39-44. 